\title{
Philosophiques
}

Marie Garrau, Politiques de la vulnérabilité, Paris, CNRS

Éditions, 2018, 358 pages

\section{Cécile Gagnon}

Volume 45, numéro 2, automne 2018

URI : https://id.erudit.org/iderudit/1055284ar

DOI : https://doi.org/10.7202/1055284ar

Aller au sommaire du numéro

Éditeur(s)

Société de philosophie du Québec

ISSN

0316-2923 (imprimé)

1492-1391 (numérique)

Découvrir la revue

Citer ce compte rendu

Gagnon, C. (2018). Compte rendu de [Marie Garrau, Politiques de la

vulnérabilité, Paris, CNRS Éditions, 2018, 358 pages]. Philosophiques, 45(2),

551-555. https://doi.org/10.7202/1055284ar d'utilisation que vous pouvez consulter en ligne.

https://apropos.erudit.org/fr/usagers/politique-dutilisation/ 
requiert une maîtrise technique hautement artificielle. Dans son ambiguité, elle fait signe vers la distinction subtile mais cruciale entre envoûtement et enchantement. Là où l'envoûtement nous coupe des émotions et du corps par une déréalisation hallucinée, l'enchantement alimente le rêve d'une autre modalité d'action que le mouvement mécanique, que la reproduction du monde à l'identique.

En proposant une relecture des événements du I 3 novembre 2015 à Paris, le dernier essai du volume tente une actualisation de la dialectique du son, tout à la fois promesse de jouissance et signe annonciateur de la terreur. E. Buch se demande ainsi ce qui, des rythmes rock, des hurlements des armes ou des sirènes des véhicules d'État, représente une issue hors du monde administré. Toutes, en réalité, sont ambigües, comme l'étaient naguère le chant des sirènes pour Ulysse: chant de mort et chant de jouissance, présence mythique à l'intérieur de la raison qu'elle échoue à conjurer par ses propres moyens, parce qu'elle se croit radicalement autre que lui.

Pluriel et riche, ce volume, on l'a vu, manifeste la grande diversité de voies possibles pour une théorie critique contemporaine soucieuse de sa tradition, dont l'un des jalons, et non des moindres, reste ce conte moderne qu'est la Dialectique de la Raison. Par la multiplicité des interprétations qu'ils suscitent, les textes d'Adorno et de Horkheimer nous offrent encore des armes pour comprendre notre présent.

AURÉLIA PEYRICAL

Université Paris-Nanterre

\section{Marie Garrau, Politiques de la vulnérabilité, Paris, CNRS Éditions, 2018, 358 pages}

Dans son ouvrage "Politiques de la vulnérabilité », la philosophe Marie Garrau présente un projet politique clair qui prend acte de la vulnérabilité fondamentale des sujets et qui valorise une forme d'autonomie relationnelle telle que celles qui sont mises en avant dans l'éthique du care de Joan Tronto et dans la théorie de la reconnaissance d'Axel Honneth. Ainsi, Garrau souhaite montrer que seule une société qui garantit à ses citoyennes et citoyens - à travers une politique de la vulnérabilité - l'accès aux conditions relationnelles et sociales de leur autonomie peut être dite «juste» (Garrau, 20I 8, I7).

L'autrice nous propose, pour ce faire, une analyse de travaux variés portant sur la vulnérabilité. L'aspect pluridisciplinaire de cette démarche est l'un des éléments qui rend cet ouvrage particulièrement pertinent, puisqu'en faisant dialoguer des travaux de philosophie morale et politique ainsi que des travaux de sociologie, Garrau parvient à présenter les éthiques du care non pas comme un courant féministe évoluant parallèlement aux débats 
politiques contemporains, mais bien comme étant au cœur des discussions actuelles en philosophie sociale et politique.

Dans la première partie de son ouvrage, Garrau se concentre sur trois approches philosophiques de la vulnérabilité: celles de Martha Nussbaum, de Joan Tronto et d'Axel Honneth. Elle souhaite ainsi montrer qu'il existe deux niveaux de vulnérabilité: I) la vulnérabilité fondamentale, dimension constitutive de l'existence humaine qui est à la fois inévitable et indépassable, mais pourtant trop souvent niée; et 2) les vulnérabilités problématiques, produites selon les contextes inégalitaires et les structures de domination dans lesquels évoluent les sujets.

L'autrice revient tout d'abord sur les travaux de Nussbaum dans lesquels la vulnérabilité fondamentale est décrite comme un «état d'ouverture et d'exposition à des évènements que nous ne maitrisons pas, qui procèdent des dimensions corporelles et temporelles de notre existence et de notre dépendance à notre environnement extérieur» (Garrau, 20I8, 27). Toutefois, si les travaux de Nussbaum permettent de bien saisir l'importance de la vulnérabilité fondamentale, Garrau souligne par ailleurs les limites de l'approche des capabilités qu'elle propose lorsqu'il est question d'apporter une réponse politique à la vulnérabilité.

Garrau montre ensuite que l'éthique du care de Tronto permet de voir la vulnérabilité non pas comme l'opposé de l'autonomie, mais bien comme son nécessaire corrélat (Garrau, 20I 8, 68). Étant toutes et tous des sujets fondamentalement vulnérables, nous dépendons constamment du care des autres. Dès lors, l'autonomie «se développe toujours sur fond de vulnérabilité» dans des dynamiques d'interdépendance avec les autres. Ainsi, la dépendance n'est pas un frein à l'autonomie, mais bien sa condition de possibilité. Néanmoins, si la vulnérabilité fondamentale n'est pas un problème en soi, il existe d'un autre côté des vulnérabilités problématiques qui surviennent lorsque des sujets cessent d'être l'objet d'attention et de care de la part des autres.

L'autrice propose donc de compléter la thèse de Tronto en y articulant la théorie de la reconnaissance de Honneth, pour qui l'absence de reconnaissance positive produit des «identités fragilisées» qui, en fonction du contexte dans lesquelles elles surviennent, peuvent donner lieu à différentes formes de vulnérabilité (Garrau, 20 I 8, I08). Dès lors, la justice d'une société dépend "de sa capacité à assurer à ses membres des conditions de reconnaissance mutuelle [c'est-à-dire] à instaurer des formes d'intégration sociale qui promeuvent l'autoréalisation individuelle de manière égale» (Garrau, 2018, I I4).

En faisant la synthèse de ces trois approches philosophiques, Garrau montre que la vulnérabilité fondamentale des sujets n'est pas simplement due à la dimension corporelle de l'existence, mais également au contexte social et relationnel dans lequel ils et elles évoluent. Ainsi, l'autrice nous invite à revoir notre conception rationaliste et individuelle de l'autonomie au profit d'une autonomie comprise comme "un accomplissement fragile, 
qui repose sur des conditions relationnelles et sociales nombreuses, au centre desquelles se tiennent différentes formes de reconnaissance » (Garrau, 20I8, I43). Cette conception relationnelle de l'autonomie permet de la penser à partir de la vulnérabilité fondamentale. Dès lors, les vulnérabilités problématiques - c'est-à-dire qui ne découlent pas des dimensions corporelles et relationnelles de l'existence - ressortent, et il apparait essentiel d'offrir une réponse politique appropriée à ces dernières en transformant les contextes et les processus sociaux qui les causent.

Dans la deuxième partie de son ouvrage, Garrau présente trois approches sociologiques de la vulnérabilité, soit celles de Robert Castel, de Serge Paugman et de Colette Guillaumin. Elle montre dans un premier temps que la vulnérabilité est notamment fonction des liens qui rattachent un sujet à des collectifs. L'approche de la vulnérabilité problématique proposée par Castel, c'est-à-dire comprise comme un problème de désaffiliation, lui permet de concevoir la vulnérabilité ni comme une question de subordination ni comme une question de pauvreté, mais bien comme une inscription dans des relations sociales instables et fragiles (Garrau, 201 8, I78).

Par la suite, Garrau vient approfondir l'approche de Castel en y ajoutant le concept de disqualification sociale développé par Paugman, selon lequel la conscience de la vulnérabilité constitue en soi «un élément central du devenir vulnérable» (Garrau, 2018, 21 5).

Finalement, l'autrice articule à ces deux théories les travaux de Guillaumin, qui développa le concept de «sexage» pour dénoncer le fait que, dans les sociétés occidentales modernes, l'autonomie des hommes s'est construite par le biais de l'appropriation du temps, des corps et du produit des corps des femmes. Cette domination de genre s'exerçant par le biais de normes et de pratiques sociales, et justifiée symboliquement par l'idée d'une nature "distincte", a favorisé la dépendance financière des femmes et fut conséquemment un frein au développement de leur autonomie. Ce contexte social inégalitaire a donc rendu les femmes, en tant que groupe, particulièrement vulnérables. Encore une fois, Garrau montre la complémentarité des trois approches présentées dans cette partie et en offre une synthèse claire et cohérente avec la conception relationnelle de l'autonomie défendue dans les chapitres précédents.

Dans la troisième et dernière partie de son ouvrage, Garrau propose une politique de la vulnérabilité en s'appuyant sur le républicanisme contemporain. Elle présente tout d'abord l'idéal de non-domination de Philip Pettit. L'autrice montre que cet idéal, qui comporte une dimension intersubjective importante, est beaucoup plus complet que le concept classique de liberté comme non-interférence (la liberté négative), puisqu'il reconnaît la vulnérabilité fondamentale des citoyennes et citoyens ainsi que leur dépendance mutuelle. Garrau soutient qu'il est par ailleurs nécessaire d'élargir la conception de la non-domination de Pettit afin de faire de l'autonomie une condition nécessaire à la liberté. 
Or, si la promotion de l'autonomie — passant notamment par l'accès à la délibération publique - devient de la sorte centrale, il apparaît dès lors inévitable selon Garrau d'intégrer au républicanisme l'apport des théories du care et de la reconnaissance. En effet, au delà de la démocratie de la contestation proposée par Pettit, l'autrice prend acte des limites de la conception classique de la délibération énoncée dans les travaux de Lynn Sanders et Iris Young, et plaide en faveur d'une démocratie participative au sein de laquelle les normes de délibération et de communication seraient élargies de façon à les rendre plus inclusives, afin que les plus vulnérables puissent y faire entendre leur voix (Garrau, 2018, 300). Une telle participation permettrait non seulement une autonomisation des citoyennes et citoyens qui y prennent part, mais également, par la confrontation des points de vue et la compréhension des situations des autres, l'instauration d'une reconnaissance mutuelle.

S'inspirant des travaux du Stuart White, Garrau plaide finalement en faveur de l'instauration d'un État social garantissant: I) un revenu minimal d'existence; 2) des opportunités égales d'accès à l'éducation et à l'emploi; et 3) la promotion (notamment à travers l'éducation) des valeurs de care et la reconnaissance mutuelle des citoyennes et citoyens afin de favoriser le développement de leur autonomie.

Si cette dernière section est fort intéressante en raison de la présentation de mesures concrètes pour mettre en place une politique de la vulnérabilité, Garrau défend une conception de la démocratie plus participative et contestataire qui semble se déployer à l'intérieur des frontières classiques de la sphère publique. En effet, l'autrice ne questionne pas de front la distinction entre sphère publique et sphère privée, distinction qui est pourtant en grande partie responsable de la dévalorisation du travail du care, ainsi que des prestataires et des bénéficiaires de soins. Bien qu'il soit essentiel que les institutions publiques deviennent des lieux d'apprentissage des vertus, qu'elles valorisent le travail du care et mettent en avant une conception de l'autonomie qui reconnaît la vulnérabilité de toutes et tous, l'un des apports importants des travaux de Tronto a été de montrer que prendre part au travail du care - notamment dans la sphère privée - est un élément essentiel pour reconnaître sa vulnérabilité et sa dépendance aux autres et ainsi développer des dispositions essentielles à une éthique du care, telles que l'attention aux autres et la sollicitude. Ainsi, il aurait été intéressant que Garrau montre comment une politique de la vulnérabilité pourrait redéfinir la justice non seulement au sein des institutions publiques, mais également au cœur de la sphère privée.

Cela étant, cet ouvrage demeure grandement pertinent puisque Garrau réussit non seulement à y présenter une quantité impressionnante de théories traitant de la vulnérabilité, mais surtout à les articuler les unes aux autres afin d'en faire ressortir un projet politique clair et convainquant. De plus, en présentant l'éthique du care de Tronto comme une approche com- 
plémentaire de la théorie de la reconnaissance de Honneth et de différentes approches sociologiques, Garrau parvient à positionner les éthiques du care au cœur des débats contemporains en philosophie politique, ce qui renforce d'autant plus l'intérêt pour cet ouvrage.

CÉCILE GAGNON

Université Laval

Michel Foucault, Les aveux de la chair, Paris, Gallimard, 2018, 448 pages

C'est quatre décennies après la parution du premier tome de Histoire de la sexualité, de Michel Foucault (I926-I984), que le quatrième tome, Les aveux de la chair, conclut le projet colossal sur lequel le philosophe français, pourtant réfractaire à l'idée d'être publié à titre posthume, a œuvré jusqu'au crépuscule de ses jours. Comment les réflexions sur la sexualité menées par les Pères de l'Église peuvent-elles intéresser le lectorat contemporain des sociétés sécularisées? C'est ce qu'entreprendra d'exposer Foucault en déterrant les fondements théoriques qui ont mené à l'établissement de valeurs comme le culte de la vie et le mépris du corps, la soumission et la domination de la volonté (p. I 27).

En étudiant comment la patristique réfléchit sur la conduite des corps, Foucault entend montrer comment, par la direction des pensées et des désirs, de même que par l'articulation des premières en actions et comportements "adéquats ", le sujet en arrive à se révéler à lui-même et à concilier divers registres de vérité souvent en tension. C'est là l'un des paradoxes de la spiritualité chrétienne: «la véridiction de soi-même est liée fondamentalement à la renonciation à soi » (p. I45), c'est-à-dire que le soi est appelé à se renier lui-même pour arriver à correspondre, en pensées comme en actions, aux préceptes d'un idéal transcendant. C'est ainsi que l'institution religieuse est devenue un puissant outil de contrôle, dont l'une des formes les plus manifestes de l'exercice de ce biopouvoir se trouve dans la capacité de convaincre les fidèles de se reproduire ou non (p. 252, 259). Le quotidien des couples et l'organisation de la cellule familiale sont alors tombés sous la juridiction des structures étatiques et des injonctions religieuses prenant en charge la gestion du désir (p. 253).

Foucault montre, par le recours aux textes et lettres des Pères de l'Église, par quelle habile substitution le régime de l'aphrodisia (celui des plaisirs valorisés par l'Antiquité) fait place à un régime où la fonction morale du devoir conjugal (fabriquer de nouvelles âmes) subordonne l'acte sexuel à une obligation morale. Pour les chrétiens, la réflexion sur la sexualité importe parce qu'elle est le corollaire d'un principe central: le refus d'accorder une quelconque valeur à la vie matérielle. Dans cette perspective, tout élan hédoniste doit être réprimé et, dans l'éventualité où cela s'avèrerait 